\title{
Survey of the use of rapid sequence induction in the accident and emergency department
}

\author{
A Walker, J Brenchley
}

\begin{abstract}
Objectives-To determine the current position regarding the use of rapid sequence induction (RSI) by accident and emergency (A\&E) medical staff and the attitudes of consultants in $A \& E$ and anaesthetics towards this.

Methods-A questionnaire was designed that was distributed to consultant anaesthetists and $A \& E$ physicians in hospitals receiving over 50000 new $A \& E$ patients per year.

Results-A total of 140 replies were received (a response rate of $72 \%$ ). The breakdown of results is shown. There was wide difference of opinion between anaesthetists and $A \& E$ consultants as to who performs RSI at present in their $A \& E$ departments, however two thirds of anaesthetists thought $A \& E$ staff with appropriate training and support should attempt RSI either routinely or in certain circumstances.

Conclusions-A\&E staff in several hospitals routinely undertake RSI and the majority of $A \& E$ consultants thought that RSI would be undertaken by $A \& E$ staff if an anaesthetist were unavailable. There is disagreement regarding the length of anaesthetic training required before $A \& E$ medical staff should undertake RSI.

(F Accid Emerg Med 2000;17:95-97)
\end{abstract}

Keywords: rapid sequence induction; anaesthesia; training

The definitive method of securing the airway of patients in the accident and emergency (A\&E) department involves placement of a cuffed endotracheal tube. In most critically ill medical and trauma patients this is best achieved using rapid sequence induction (RSI). This procedure involves intravenous induction and paralysing agents and the application of cricoid pressure as described in standard protocols. RSI is usually undertaken by the on-call anaesthetic team. ${ }^{1}$ Such support is usually readily available to $\mathrm{A} \& \mathrm{E}$ departments but occasions may arise when an anaesthetist is not able to attend immediately.

Some A\&E physicians have been using the technique for a number of years both in the hospital $^{2}$ and pre-hospital environment. ${ }^{3} \mathrm{Re}-$ cently there has been interest in the more widespread practice of this technique by $\mathrm{A} \& \mathrm{E}$ staff and it has been suggested that senior emergency department staff should be competent in RSI after a suitable period of training.
Emergency physicians in the United States and Australia regard RSI as the cornerstone of airway management in the emergency department, ${ }^{4}$ with no evidence of adverse outcome for patients. ${ }^{56}$

The aim of our study was to determine attitudes of consultants in both anaesthesia and $\mathrm{A} \& \mathrm{E}$ to the use of the technique by $\mathrm{A} \& \mathrm{E}$ staff.

\section{Method}

A questionnaire was devised to assess current practice and opinion regarding the use of RSI in A\&E departments. Using the 1996 BAEM directory, hospitals with more than 50000 new $A \& E$ attendances per year were identified. The questionnaire was sent to the A\&E and anaesthetic departments in each of 97 hospitals. It was addressed to the consultant responsible for liaison with $\mathrm{A} \& \mathrm{E} /$ anaesthetics respectively. As it became apparent that not all questionnaires reached their destination a further copy was sent. The questionnaire included a brief description of RSI and the questions were as the headings in the results section below.

\section{Results}

In total 140 replies were received, 71 from anaesthetists and 69 from A\&E consultants (a response rate of $72 \%$ ). Replies from both anaesthetic and $\mathrm{A} \& \mathrm{E}$ consultants were received from 50 hospitals.

Who undertakes RSI in your department? In answer to this question the anaesthetists replied that $93 \%$ of the time it was anaesthetic staff only and $7 \%$ of the time anaesthetic and $\mathrm{A} \& \mathrm{E}$ staff. The corresponding figures for the A\&E consultants were $69 \%$ and $31 \%$.

In hospitals where replies were received from both departments $74 \%$ showed agreement between departments: $6 \%$ thought A\&E would perform RSI, $68 \%$ thought they would not. In $26 \%$ of these hospitals there was a difference of opinion: in $4 \%$ the anaesthetist thought $\mathrm{A} \& \mathrm{E}$ performed RSI but A\&E thought they would not, and in $22 \%$ the $\mathrm{A} \& \mathrm{E}$ consultant thought they would, but the anaesthetist thought they would not.

Grade of anaesthetist crash bleeped to the $A \mathcal{E} E$ department

The grade of anaesthetist crash bleeped to the A\&E department is shown in table 1.

\section{Would A\&E staff attempt RSI?}

When asked whether A\&E staff would attempt RSI if an anaesthetist were unavailable $69 \%$ of A\&E consultants thought they would compared with only $34 \%$ of anaesthetic consultants. 
Table 1 Grade of anaesthetist crash bleeped to AE E (\%)

\begin{tabular}{lcc}
\hline & $\begin{array}{l}\text { Anaesthetist } \\
\text { replies }\end{array}$ & $\begin{array}{l}\text { A } E \text { E } \\
\text { replies }\end{array}$ \\
\hline Senior house officer & 33 & 23 \\
Specialist registrar & 49 & 58 \\
Staff grade & 4 & 2 \\
Senior registrar & 2 & 1 \\
Consultant & 12 & 16 \\
\hline
\end{tabular}

Where replies were received from both departments within a hospital $28 \%$ agreed that A\&E would attempt RSI, $20 \%$ agreed they would not. In $8 \%$ of hospitals the anaesthetist thought $\mathrm{A} \& \mathrm{E}$ would attempt it but the $\mathrm{A} \& \mathrm{E}$ consultant thought they would not, and in $36 \%$ of hospitals the A\&E consultant thought they would attempt RSI but the anaesthetist thought they would not.

Grade of $A \mathcal{E} E$ staff who at present attempt RSI The grade of $A \& E$ staff who at present attempt RSI is shown in table 2 .

\section{Are AEE specialist registrars resident?}

When asked if $\mathrm{A} \& \mathrm{E}$ specialist registrars were resident $81 \%$ of respondents believed an $\mathrm{A} \& \mathrm{E}$ specialist registrar to be resident in the department but not throughout the 24 hour period: $68 \%$ of anaesthetists thought registrars were resident overnight compared with the 37\% stated by A\&E consultants.

\section{Is an operating department assistant (ODA)} called to AEE for RSI?

In answer to this question $65 \%$ of anaesthetists and $49 \%$ of $\mathrm{A} \& \mathrm{E}$ consultants thought an ODA would be called to $A \& E$ when rapid sequence induction is undertaken.

How much anaesthetic training would be required before $A \mathcal{E} E$ specialist registrars could undertake RSI?

Responses to this question are shown in table 3.

Given appropriate training and support, should an $A \mathcal{E} E$ specialist registrar undertake RSI? In answer to the above question $20 \%$ of the anaesthetists said yes, $44 \%$ said yes in certain circumstances, and $36 \%$ said no. The corresponding results for the $\mathrm{A} \& \mathrm{E}$ consultants were yes $38 \%$, in certain circumstances $46 \%$, and no $16 \%$.

Table 2 Grade of AE E staff who at present attempt RSI (\%)

\begin{tabular}{llllll}
\hline & $\begin{array}{l}\text { Senior } \\
\text { house } \\
\text { officer }\end{array}$ & $\begin{array}{l}\text { Specialist } \\
\text { registrar }\end{array}$ & $\begin{array}{l}\text { Senior } \\
\text { registrar }\end{array}$ & $\begin{array}{l}\text { Staff } \\
\text { grade }\end{array}$ & Consultant \\
\hline Anaesthetic replies & - & 52 & - & 8 & 40 \\
A\&E replies & 1 & 44 & 3 & 8 & 44 \\
\hline
\end{tabular}

Table 3 Time required for anaesthetic training before AE E specialist registrars could undertake RSI (\%)

\begin{tabular}{lllllllll}
\hline & & & & & \multicolumn{3}{c}{ Primary } \\
& 1 Month & 3 Months & 6 Months & 1 Year & 2 Years & FRCA & FRCA & Never \\
\hline Anaesthetic replies & 0 & 29 & 23 & 21 & 6 & 6 & 5 & 10 \\
A\&E replies & 6 & 42 & 27 & 11 & 2 & 0 & 2 & 10 \\
\hline
\end{tabular}

FRCA $=$ Fellow of the Royal College of Anaesthetists

\section{Discussion}

There was a good response rate to this questionnaire on the attitudes and beliefs of $\mathrm{A} \& \mathrm{E}$ and anaesthetic consultants to the practice of RSI by A\&E staff. The questionnaire was distributed on the basis of new $A \& E$ attendances, to assess the practices in departments in which there would be likely to be junior, middle grade, and senior cover.

Most of the data are self evident and do not need lengthy discussion. There may be differences between beliefs and actual practice. The concerns of both groups are highlighted.

As expected, most RSIs in the A\&E department are carried out at present by anaesthetists. Clearly there are a number of hospitals where the anaesthetic team are unaware that RSI may be undertaken by A\&E staff. In the majority of departments anaesthetic help is available in less than five minutes. In some cases, however, there is an unacceptable delay and training resident $\mathrm{A} \& \mathrm{E}$ staff to perform RSI may be of benefit.

It is interesting that two thirds of $\mathrm{A} \& \mathrm{E}$ departments appear to be willing to undertake RSI when no anaesthetic help is available. Only one third of anaesthetic replies considered this a possibility. Generally it was considered that only middle and senior grades would undertake RSI. A senior house officer post in $A \& E$ is often part of a junior anaesthetic trainee's curriculum. As such they would be appropriately trained to undertake the technique and may be a useful liaison between anaesthetic and $A \& E$ departments.

Although $80 \%$ of respondents have specialist registrars in their departments, in only one third of departments is such cover available throughout the 24 period. If $\mathrm{A} \& \mathrm{E}$ is to provide advanced emergency airway management staff must be available within minutes. Consideration must therefore be given to $\mathrm{A} \& \mathrm{E}$ middle grades being resident on site.

It is surprising that in only two thirds of cases of RSI in A\&E performed by anaesthetists an ODA is called.

One of the most contentious issues is the amount of training necessary to undertake RSI. Anaesthetic trainees usually perform on call duties alone after a minimum of three months supervision. In our survey there was a wide range of responses. In A\&E specialist registrar training programmes, a three month training period in anaesthetics is routinely completed: $52 \%$ of A\&E consultants and $71 \%$ of anaesthetists think this is inadequate. In contrast $75 \%$ of $\mathrm{A} \& \mathrm{E}$ replies and $52 \%$ of anaesthetic replies thought that six months' training would be sufficient. There may be a place for specific courses in RSI for A\&E staff to supplement standard training, similar to those already provided in the United States. Residency programmes in the USA now routinely include training in $\mathrm{RSI}^{6}{ }^{7}$

Most respondents thought that with appropriate training and support $A \& E$ staff should perform RSI, either routinely or in certain circumstances. In the United States RSI is the cornerstone of advanced airway management in the emergency department and studies have 
shown it can be carried out safely. Kenny et al describe minor complication rates of $6 \%$ and no major complications. ${ }^{5}$ In a study of 417 RSI by Tayal et al, "major" complications were seen in $1.4 \%{ }^{6}$ Walls et al, in a series of 1288 emergency department intubations, documented an overall complication rate of $12 \%$, with a major complication rate of $3 \%$ for RSI. ${ }^{8}$ It is difficult to compare these figures with those for anaesthetists as emergency department patients represent a distinct high risk subgroup. It is clear from the comments of anaesthetists that they are concerned that critically ill patients requiring immediate airway protection are the most difficult cases to manage. These are the patients who can least afford to wait for an anaesthetist to arrive in A\&E. It seems inappropriate to suggest that A\&E staff should perform RSI only when an anaesthetist is unavailable. It has been suggested that as emergency physicians are familiar with the acutely ill patient, they are the most appropriate group to manage the airway. If the practice of RSI by A\&E staff is limited to the occasional attempt in the sickest patients skill decay will be an issue. However if $\mathrm{A} \& \mathrm{E}$ staff perform the majority of RSIs, either with or without anaesthetic support, there will be opportunity to maintain skills. There is little documented evidence of the number of RSIs anaesthetists require to maintain competence.
In other countries RSI is becoming standard emergency medicine practice. ${ }^{9}$ A\&E medicine uses techniques from many specialties and there is no fundamental reason why RSI should be limited to anaesthetic practice. Clearly further discussion is needed to take this issue forward.

We would like to thank Mr J P Sloan and Dr A McGowan for their advice.

Conflict of interest: none.

Funding: none.

1 Association of Anaesthetists of Great Britain and Ireland. The role of the anaesthetist in the emergency service. London: Association of Anaesthetists of Great Britain and Ireland, 1991.

2 McBrien ME, Pollok AJ, Steedman DJ. Advanced airway control in trauma and resuscitation. Arch Emerg Med 1992; 9:177-80.

3 Graham CA, Meyer ADM. Prehospital emergency rapid sequence induction of anaesthesia. 7 Accid Emerg Med 1997;14:219-21.

4 Rosen P, Barkin R. Emergency medicine, concepts and clinical practice. St Louis: Mosby, 1998.

5 Kenny JF, Molloy K, Pollock M, et al. Rapid sequence induction technique for orotracheal intubation of adult non-trauma patients in a community hospital setting. Ann Emerg Med 1995;25:432-3.

6 Tayal VS, Riggs RW, Marx JA, et al. Rapid-sequence intubation at an emergency medicine residency: success rate and tion at an emergency medicine residency: success rate and
adverse events during a two-year period. Acad Emerg Med 1999;6:31-7.

7 Sakles JC, Laurin EG, Rantapaa AA, et al. Airway management in the emergency department: a one-year study of 610 tracheal intubations. Ann Emerg Med 1998;31:325-32.

8 Walls RM, Vissers RJ, Sagarin MJ, et al. Emergency department intubations-final report of the National Emergency 392.

9 American College of Emergency Physicians. Policy statement on rapid sequence induction. Dallas: ACEP, 1996. 